\title{
Mycotic aneurysm of the ascending aorta following $\mathrm{CABG}$
}

\author{
M Prech, S Grajek, A Cieśliński, M Jemielity
}

\begin{abstract}
Mycotic aneurysm of the thoracic aorta is a rare and life threatening condition. Two patients are presented (both male, aged 66 and 59 years) in whom coronary artery bypass surgery was complicated by the development of a mycotic aneurysm. Fever preceded the radiological and echocardiographic signs of the aneurysm by at least several months in both cases. Blood cultures were negative for one patient and the source of Corynebacterium $s p$ infection in the other was not determined for several months. Both patients died before surgery could correct the aneurysm.

(Heart 2000;83:e3)
\end{abstract}

Keywords: mycotic aneurysm; aorta; revascularisation; surgical complications

Mycotic aneurysm is a rare but life threatening condition. Most of over 40 cases of mycotic aneurysms of the thoracic aorta reported in the literature were connected with different surgical procedures involving the cardiovascular system. ${ }^{1-11}$ A surgical reconstruction with either aortic homograft or synthetic graft insertion in combination with prolonged administration of antibiotics has lowered mortality to
$25 \% .^{36711-13}$ Early diagnosis is important for successful treatment. We present two patients in whom coronary artery bypass graft surgery was complicated by the development of mycotic aneurysm of the ascending aorta.

\section{Case 1}

In May 1995, a 66 year old man was admitted to the cardiology department with fever and cachexia; he had a history of hypertension and inferior myocardial infarction. In June 1994, he had had a multivessel revascularisation for triple vessel coronary disease, and in December 1994 he was admitted with a recurrence of fever, which had appeared for the first time two months earlier. Laboratory studies (white blood cell count (WBC) $6.3 \times 10^{9} / 1$, erythrocyte sedimentation rate (ESR) $43 \mathrm{~mm}$ in the first hour, negative blood cultures), radiological examination (normal chest roentgenogram), echocardiography (aortic root diameter $3.0 \mathrm{~cm}$ in the transthoracic assessment), endoscopy, scintigraphy, and other tests did not reveal the origin of the fever. After two weeks of antibiotics the patient was discharged afebrile and did well for three months. However, in March 1995 he was admitted with septicaemia. Corynebacterium sp (susceptible to imipenem, vancomycin, and ciprofloxacin) was isolated from blood cultures. The source of infection 1st Department of Cardiology, University School of Medical Sciences, Dluga 1/2, 61848 Poznań, Poland $M$ Prech

S Grajek

A Cieśliński

M Jemielity

Correspondence to: Dr Prech

Accepted for publication 26 August 1999

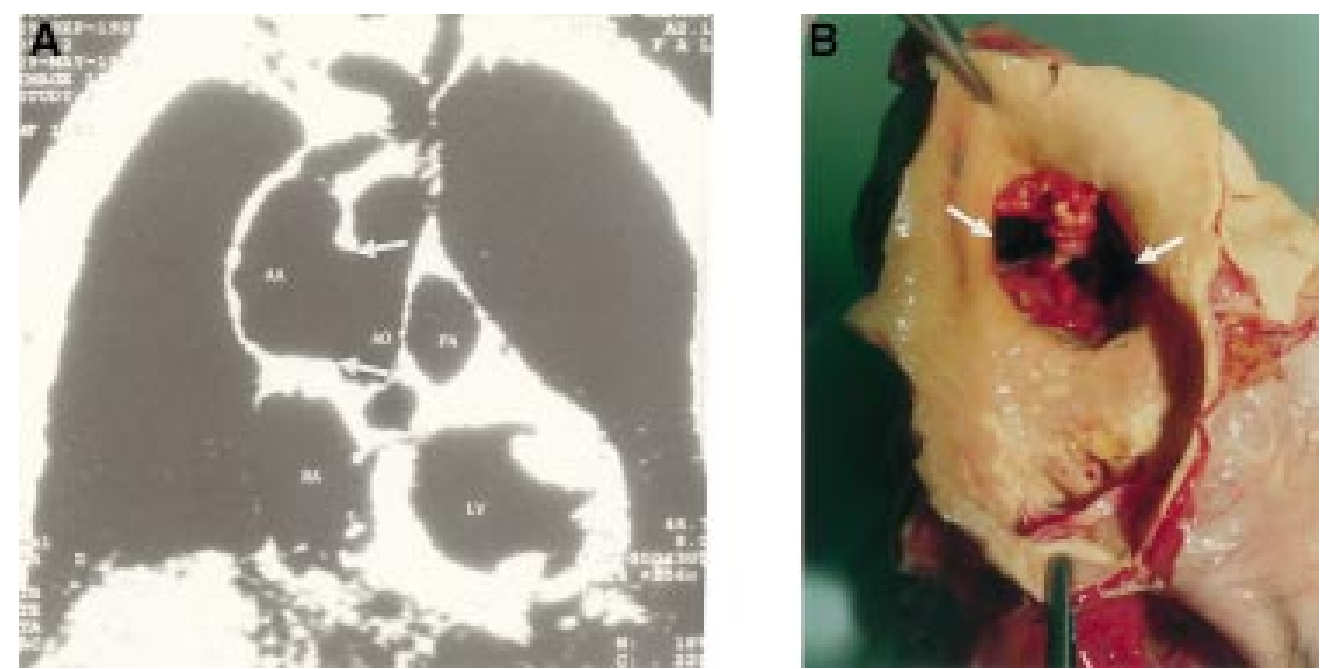

Figure 1 (A) Magnetic resonance image: a large aneurysm of the ascending aorta ( $A A)$; arrows point to the mouth of the aneurysm. (B) Aneurysm of the ascending aorta (mouth of aneurysm between arrows) at necropsy. AO, aorta; $P A$, pulmonary artery; $R A$, right atrium; LV, left ventricle. 

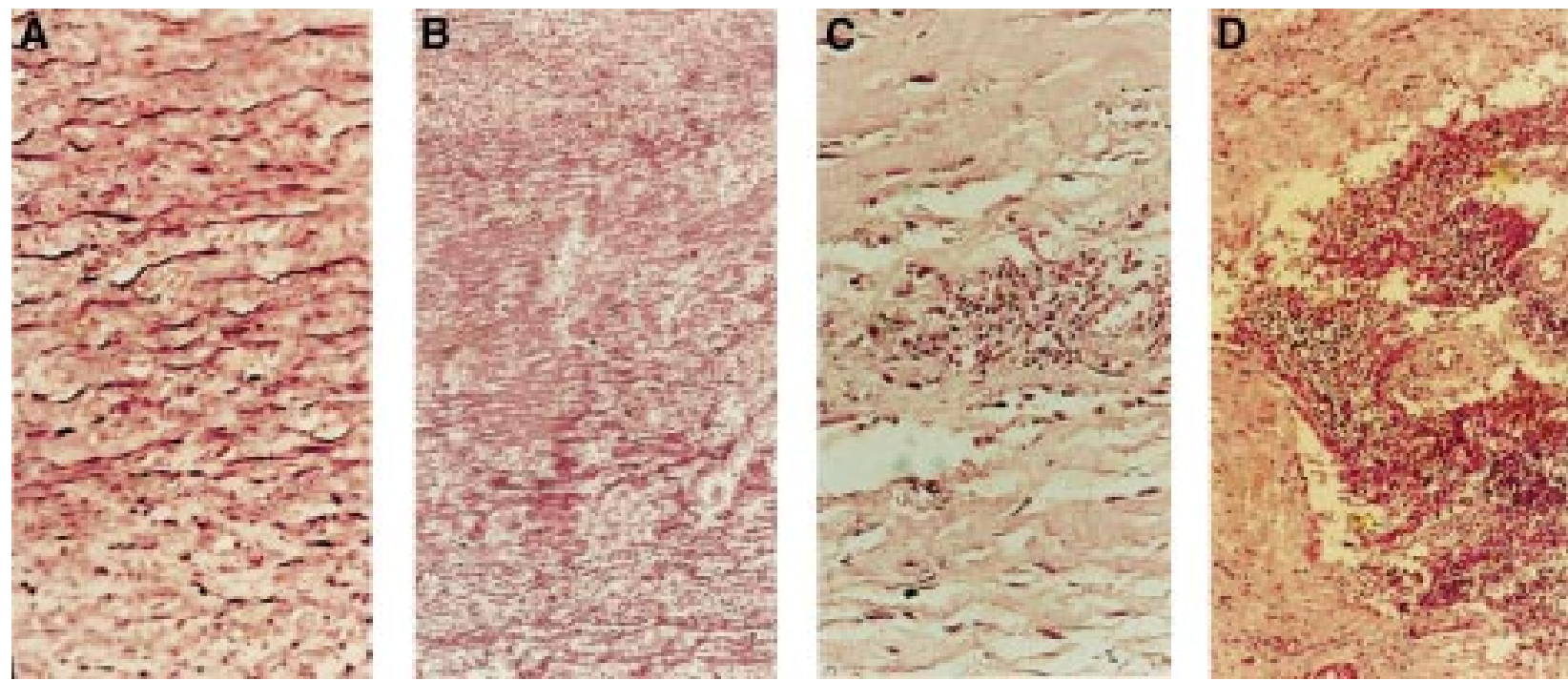

Figure 2 Longitudinal sections of the walls of the aneurysm. (A) Advanced $(\times 100)$ and $(B)$ total ( $\times 50)$ disruption of elastin lamellae (Verchoff's stain). (C) Small lymphocytic infiltration of the wall of the aneurysm $(\times 100)$. (D) Case 2: Large infiltration of the wall of the aneurysm consisting of lymphocytes, monocytes, and proliferated capillaries $(\times 50)$.

was not clear and after two months of treatment with vancomycin the surgical wires (the supposed cause of the fever) were removed from the sternum and he was moved to the cardiology department.

On admission, he was febrile and cachectic, and complaining of chest pain. Laboratory investigations showed anaemia, normal WBC count (but with a shift to the left), ESR $12 \mathrm{~mm}$ in the first hour, deteriorated parameters of renal function, and negative blood cultures. Pseudomonas aeruginosa was isolated from urine. The dimensions of the heart chambers and the aorta were normal on transthoracic echocardiography, whereas chest radiography showed a widened mediastinum. An aneurysm of the ascending aorta (presumably mycotic) was diagnosed by transoesophageal echocardiography. Magnetic resonance imaging confirmed a large $(6.0 \mathrm{~cm}$ wide, $7.2 \mathrm{~cm}$ long) aneurysm of the ascending aorta (fig 1A). Despite high doses of antibiotics the patient

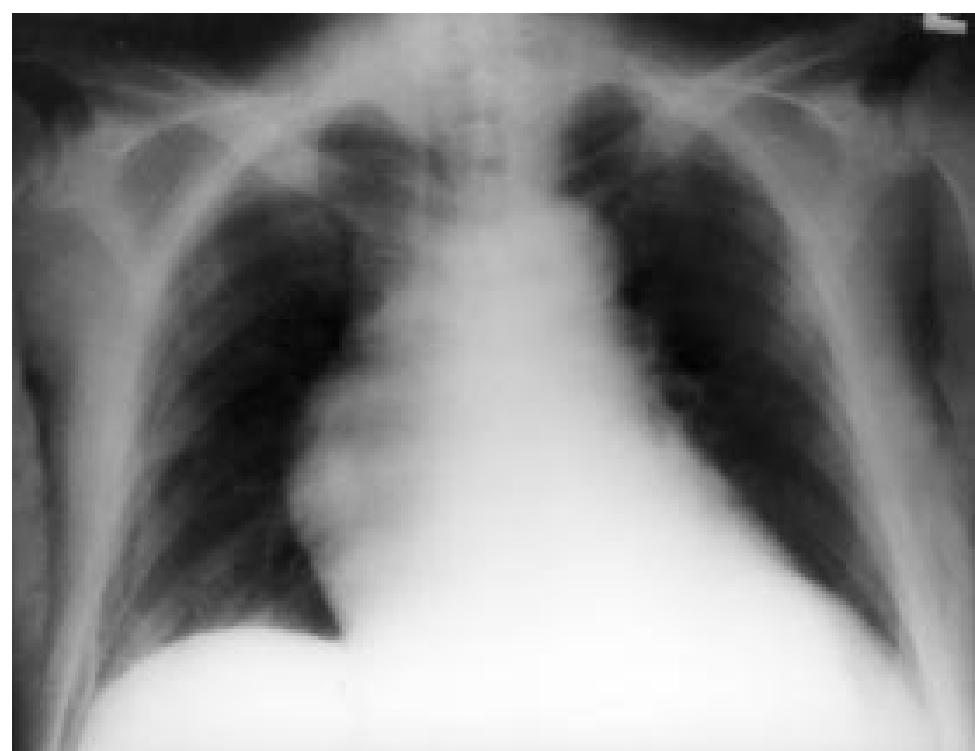

Figure 3 Chest roentgenogram showing a widened mediastinum. developed septic shock and died before surgery. An aneurysm of the ascending aorta encompassing ostia of the venous grafts was found at necropsy (fig 1B). Histological examination of the aneurysm wall tissue (covered with bacterial vegetation) revealed complete destruction of elastin fibres (fig 2A and B) and lymphocytic inflammatory infiltrations (fig $2 \mathrm{C})$.

\section{Case 2}

In May 1996 a 59 year old man was admitted to the department of cardiology because of syncope. His medical history included hyperthyreosis, hypertension, inferior myocardial infarction, and diabetes mellitus. He underwent vein bypass of the left anterior descending artery and right posterior descending artery on 11 September 1995. The same day he was reoperated owing to haemorrhage caused by the rupture of the ascending aorta. He was febrile in March 1996 and the following month endophthalmitis was diagnosed.

On admission he was blind, afebrile, and cachectic. He complained of diarrhoea. Laboratory investigations revealed anaemia, WBC $28 \times 10^{9} / 1$, ESR $76 \mathrm{~mm}$ in the first hour, and raised serum creatinine. An initial blood culture was negative. Chest radiography showed a widened mediastinum (fig 3). An aneurysm of the ascending aorta (up to $9 \mathrm{~cm}$ diameter) was observed in transthoracic echocardiography. The patient died suddenly before any further investigations or surgery could be performed. Postmortem examination showed a large aneurysm of the ascending aorta. Histological examination, similar to case 1 , showed complete destruction of elastin fibres and large inflammatory infiltrations of the aorta wall consisting of lymphocytes, monocytes, and proliferated capillaries (fig 2D).

\section{Discussion}

Mycotic aneurysm (also called infected false aneurysm or acute bacterial aortitis) is a localised abnormal dilatation of the arterial wall that 
develops secondary to an infection. ${ }^{6}{ }^{12}$ The development of primary mycotic aneurysms (not related to infective endocarditis) have been described as a complication of different surgical procedures involving the aorta. ${ }^{5}$ Single cases of the development of mycotic aneurysms following such common procedures as coronary revascularisation or percutaneous transluminal coronary angioplasty have also been reported. ${ }^{237}$

The growth of mycotic aneurysms is rapid, several centimetres per month. ${ }^{357}$ In contrast, the progress of non-mycotic aneurysms is estimated between 0.3 and $0.6 \mathrm{~cm}$ per year. ${ }^{14} 15$ Factors thought to be responsible for such rapid enlargement are the virulence of the organisms and arterial blood pressure, which provides the expanding force; however, the latter is also a factor in the development of slowly progressing non-mycotic aneurysms. ${ }^{516}$

Intimal damage, an inherent part of surgery, and infiltration of the aortic wall with bacteria and inflammatory cells triggers the process of aneurysm formation. ${ }^{716} 17$ Different inflammatory infiltrations were observed in both presented cases (fig 2C and D). Virulent bacteria - staphylococci and salmonellae most often isolated from the blood of patients diagnosed with mycotic aneurysms - usually cause an intense inflammatory reaction. ${ }^{18}$ Once started, however, further dilatation of the aortic wall might be independent of the presence of bacteria. In some cases (including case 2) less virulent organisms or no organisms are isolated. ${ }^{5}{ }^{16}$ Matrix metalloproteinases localised to inflammatory cells (mainly monocyte derived macrophages) and smooth muscle cells cause elastin and collagen breakdown (fig 2A and $\mathrm{B}$ ) and consequent dilatation of the aortic wall. ${ }^{1517}{ }^{19}$ Freestone et al suggested that the rate of aneurysm expansion may reflect the intensity of inflammation within the aortic wall. ${ }^{17}$

There are probably other factors that affect the process of aneurysm formation: the extent of the intimal damage - the more severe the destruction of the aortic wall, the easier it will be for bacteria and inflammatory cells to infiltrate; immunological disorders which enhance the development of mycotic aneurysms especially in transplant patients ${ }^{8-10}$; and pharmacological treatment-antibiotics and antiinflammatory drugs might slow the process of aneurysm enlargement. Unfortunately, a conservative approach to mycotic aneurysms cannot prevent further dilatation of the aortic wall as the damaged elastin fibres are not resynthesised in the adult aorta. ${ }^{20}$

Mycotic aneurysm of the aorta should be suspected in patients with fever of unknown origin who have undergone any invasive procedure of the cardiovascular system. However, precise diagnosis is difficult, especially in the early stages because of lack of specific symptoms. In most reported cases (including both of our patients) fever, the most common symptom of the disease, preceded radiological and echocardiographic signs of aneurysm formation by (at least) several months. ${ }^{2711}$

We conclude that in febrile patients who have undergone any invasive procedure of the cardiovascular system, meticulous investigation including transthoracic echocardiography or magnetic resonance imaging might help to diagnose the cause of the fever. Once a mycotic aneurysm is diagnosed, surgical treatment with in situ insertion of an aortic homograft or synthetic graft should be done without delay. Pharmacological treatment alone is ineffective and will not save the patient's life. ${ }^{6}{ }^{16}$

1 Murphy PD, Glazier DB, Krause TJ. Mycotic aneurysm of the thoracic aorta caused by Clostridium septicum. Ann Thorac Surg 1996;62:1835-7.

2 Crosby IK, Tegtmeyer C. Mycotic aneurysm of the ascending aorta following coronary revascularisation. Ann Thorac Surg 1978;25:474-6.

$3 \mathrm{Oz}$ MC, McNicholas KW, Serra AJS, et al. Review of salmonella mycotic aneurysms of the thoracic aorta. $\mathcal{F}$ Cardiovasc Surg 1989;30:99-103.

4 Pasic M, Carrel T, Segesser L, et al. In situ repair of mycotic aneurysm of the ascending aorta. F Thorac Cardiovasc Surg 1993; 105:321-6.

5 Vilacosta I, Bustos D, Ciguenza R, et al. Primary mycotic aneurysm of the ascending aorta diagnosed by transesophageal echocardiography. $f$ Am Soc Echocardiogr 1998; 11:216-18.

6 Meerkin D, Yinnon AM, Munter RG, et al. Salmonella mycotic aneurysm of the aortic arch: case report and review. Clin Infect Dis 1995;21:523-8.

7 Follis FM, Paone RF, Wernly JA. Mycotic aneurysm of the ascending aorta after coronary revascularisation. Ann Thorac Surg 1994;58:236-8.

8 McGiffin DC, Galbraith AJ, McCarthy JB, et al. Mycotic false aneurysm of the aortic suture line after heart transplantation. $\mathcal{F}$ Heart Lung Transplant 1994;13:926-8.

9 Thomson D, Menkis A, Pflugfelder P, et al. Mycotic aortic aneurysm after heart-lung transplantation. Transplantation 1989;47:195-7

10 Albes J, Haverich A, Freihorst J, et al. Management of mycotic rupture of the ascending aorta after heart-lung transplantation. Ann Thorac Surg 1990;50:982-3.

11 Chen YF, Lin PY, Yen HW, et al. Double mycotic aneurysms of the ascending aorta. Ann Thorac Surg 1997;63:529-31.

12 Pasic MD. Mycotic aneurysm of the aorta: evolving surgical concept. Ann Thorac Surg 1996;61:1053-4.

13 Knossala C, Weng Y, Yankah AC, et al. Using aortic allograft material to treat mycotic aneurysms of the thoracic aorta. Ann Thorac Surg 1996;61:1146-52.

14 Dapunt OE, Galla JD, Sadeghi AM, et al. The natural history of thoracic aortic aneurysms. $\mathcal{F}$ Thorac Cardiovasc Surg 1994;107:1323-33.

15 Shah PK. Inflammation, metalloproteinases, and increased proteolysis. An emerging pathophysiological paradigm in aortic aneurysm. Circulation. 1997;96:2115-17.

16 Scheld WM, Sande MA. Cardiovascular infections. In: Mandell GL, Bennett JE, Dolin R, eds. Principles and practice of infectious diseases. 4th ed. New York: Churchill Livingstone, 1995:769-77.

17 Freestone T, Turner RJ, Coady A, et al. Inflammation and matrix metalloproteinases in the enlarging abdominal aortic aneurysm. Arterioscler Thromb Vasc Biol 1995;15:1145tic and.

18 Berkmen YM. Medical aspects of infectious aortitis. In: Lande A, Berkmen YM, McAllister HA, eds. Aortitis. Clinical, pathologic, and radiographic aspects. New York: Raven cal, pathologic, and radiog

19 Zatina MA, Zarins CK, Gewertz BL, et al. Role of medial lamellar architecture in the pathogenesis of aortic aneurysms. F Vasc Surg 1984;1:442-8.

20 MacSweeney STR, Powell JT, Greenhalgh RM. Pathogenesis of abdominal aortic aneurysm. Br F Surg 1994;81:93541 . 